\title{
INFLUÊNCIA DE PARÂMETROS OPERACIONAIS NO PROCESSO DE ELETRODEPOSIÇÃO DE LIGAS Zn-Ni
}

\author{
C. Z. CASTELLI ${ }^{1}$, C. A. SIVIERO FILHO ${ }^{1}$, A. F. de ALMEIDA NETO ${ }^{1}$ \\ ${ }^{1}$ Universidade Estadual de Campinas, Faculdade de Engenharia Química \\ E-mail para contato: camilazcastelli@gmail.com
}

\begin{abstract}
RESUMO - Neste trabalho foi realizado um estudo no qual se verificou a influência da variação de parâmetros para o processo de eletrodeposição da liga metálica $\mathrm{Zn}-\mathrm{Ni}$, avaliando a eficiência de deposição em substrato de cobre. Os parâmetros estudados foram agitação mecânica e densidade de corrente elétrica, em 7 experimentos realizados conforme um planejamento fatorial $2^{2} \operatorname{com} 3$ pontos centrais, sendo as concentrações de componentes do banho eletrolítico fixas, $\mathrm{pH}$ 6,0 e temperatura ambiente. De acordo com o estudo estatístico realizado, a variável que apresentou maior influência estatisticamente significativa na eficiência de deposição foi a densidade de corrente elétrica. O modelo construído para o processo apresentou coeficiente de regressão $\left(\mathrm{R}^{2}\right)$ de 0,9486 . O depósito obtido apresentou brilho e aderência nas bordas do substrato, com eficiência de deposição de 93,04\%, e os parâmetros utilizados para sua obtenção foram agitação mecânica $15 \mathrm{rpm}$ e densidade de corrente elétrica $50 \mathrm{~mA} / \mathrm{cm}^{2}$.
\end{abstract}

\section{INTRODUÇÃO}

De acordo com os autores Hegde et al. (2010), Short et al. (1996), Ramanauskas (1999), Fratesi e Roventi (1996), Anicai et al. (1992), as ligas metálicas formadas por eletrodeposição de zinco-níquel têm sido bastante estudadas e despertam o interesse desde os anos 90, pois apresentam significante melhoria das propriedades mecânicas como dureza e ductibilidade, além de serem mais estáveis termicamente e até 6 vezes mais resistentes à corrosão quando comparadas com depósitos de zinco puro. Os revestimentos com esta liga são expressivos na indústria automotiva, e também são empregados em indústrias eletrônicas. (Riedel, 1988; Crotty, 1996 e Brooman, 1993) As ligas Zn-Ni tem sua importância ainda na substituição de ligas tóxicas de cádmio, segundo Alfantazi (1996), Wright (1994), Lin e Selman (1993) e Miyoshi (1991).

A eletrodeposição de uma liga metálica, que acontece a partir de complexos eletrolíticos, geralmente, é afetada pelo tipo de banho eletrolítico e também pelos parâmetros operacionais (Beltowska-Lehman e Indyka, 2012). Dentre os componentes aditivos do banho eletrolítico, o citrato é usado como complexante, e tem a função de estabilizar as condições de eletrodeposição e conduzir uma deposição homogênea e de boa qualidade (Gomez et al., 2001 e Beltowska-Lehman et al., 1998). Os parâmetros operacionais podem ser agitação mecânica, densidade de corrente elétrica, temperatura, aplicação de campo magnético, dentre outros. $\mathrm{O}$ presente trabalho apresenta um estudo cuidadoso da formação da liga $\mathrm{Zn}-\mathrm{Ni}$, mediante variação de agitação mecânica e densidade de corrente elétrica. 


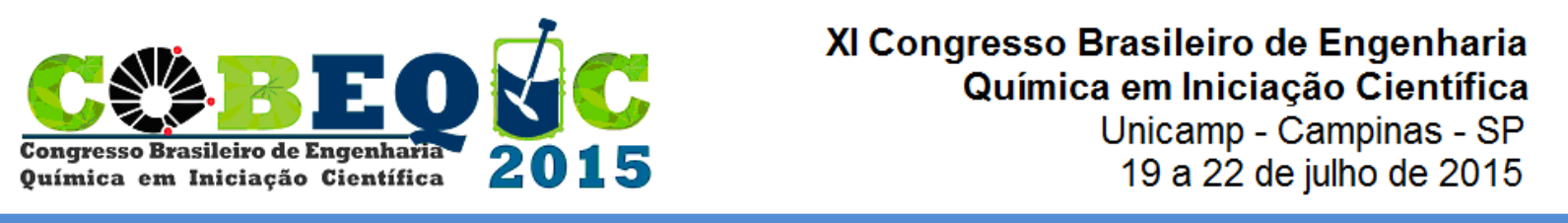

\section{PROCEDIMENTO EXPERIMENTAL}

No presente trabalho, o substrato utilizado (cátodo) foi uma placa de cobre previamente tratada, uma malha cilíndrica de platina foi utilizada como contraeletrodo e o banho eletrolítico com componentes e concentrações específicas, contendo complexos de $\mathrm{Zn}$ e $\mathrm{Ni}$ como ânodos.

O tratamento do substrato de cobre foi realizado utilizando lixas de granulação 220 e 320, respectivamente. Após o polimento, o substrato foi tratado quimicamente em ácido sulfúrico $1 \%$ e em seguida em hidróxido de sódio $10 \%$. A superfície do substrato foi lavada com água deionizada, e em seguida, aplicou-se esmalte incolor na haste para evitar que a deposição acontecesse externamente à área quadrada de $4 \mathrm{~cm}^{2} \mathrm{em}$ cada lado.

$\mathrm{Na}$ preparação do banho eletrolítico, foram usados reagentes com elevado grau de pureza analítica, e água destilada e deionizada. O banho eletrolítico foi constituído dos seguintes reagentes: $\mathrm{ZnSO}_{4} 0,1 \mathrm{~mol} / \mathrm{L}$, fonte de $\mathrm{Zn} ; \mathrm{NiSO}_{4} 0,1 \mathrm{~mol} / \mathrm{L}$, fonte de $\mathrm{Ni}$; $\left(\mathrm{NH}_{4}\right)_{2} \mathrm{C}_{6} \mathrm{H}_{6} \mathrm{O}_{7} 0,2 \mathrm{~mol} / \mathrm{L}$, agente complexante do $\mathrm{Zn}$ e Ni; $\mathrm{Na}_{2} \mathrm{~B}_{4} \mathrm{O}_{7} 3,75 \cdot 10^{-2} \mathrm{~mol} / \mathrm{L}$, utilizado no banho como agente amorfizante; $\left(\mathrm{NH}_{4}\right)_{2} \mathrm{SO}_{4} 0,13 \mathrm{~mol} / \mathrm{L}$, usado para dar maior estabilidade à solução; 1-dodecilsulfato-Na $1,04.10^{-4} \mathrm{~mol} / \mathrm{L}$ age como surfactante, acelerando o desprendimento de $\mathrm{H}_{2}$ liberado durante a deposição para evitar a formação de bolhas na liga aderida. $\mathrm{O}$ pH do banho foi ajustado adicionando-se ácido nítrico ou hidróxido de amônio.

A razão entre a carga elétrica utilizada $\left(Q_{u}\right)$ e a carga total $(Q)$ presente no sistema forneceu a eficiência de deposição do processo, dada pela Equação 1:

$$
y=\frac{Q_{u}}{Q} \cdot 100
$$

sendo, y a eficiência de deposição dada em termos percentuais (\%).

\subsection{Planejamento experimental e especiação metálica}

Tendo em vista a avaliação quantitativa da influência das variáveis de entrada sobre a eficiência de deposição do sistema, assim como suas interações, realizou-se um planejamento fatorial (Barros Neto et al., 1996) 22, com 3 experimentos no ponto central, totalizando 7 experimentos, que foram realizados em ordem aleatória para evitar erros sistemáticos. A análise estatística dos dados experimentais foi realizada com pelo software STATISTICA 7. Foram avaliados dois níveis diferentes $(-1$ e +1$)$ de agitação mecânica e densidade de corrente elétrica (I). Os resultados dos experimentos do ponto central foram incluídos na análise estatística. Os valores das variáveis de entrada estão dispostos na Tabela 1.

Tabela 1 - Agitação mecânica e densidade de corrente elétrica (I) utilizadas.

\begin{tabular}{cccc} 
Variáveis $^{\text {Níveis }}$ & -1 & 0 & +1 \\
\hline $\mathrm{I}\left(\mathrm{mA} / \mathrm{cm}^{2}\right)$ & 10 & 30 & 50 \\
\hline Agitação $(\mathrm{rpm})$ & 15 & 30 & 45 \\
\hline
\end{tabular}


Para determinação do $\mathrm{pH}$ do banho, foi realizado um estudo de especiação química dos componentes do banho. Na Figura 1 são mostrados os complexos que podem ser formados a diferentes valores de $\mathrm{pH}$.

Figura 1 - Complexos formados com íons de citrato em diferentes valores de $\mathrm{pH}$

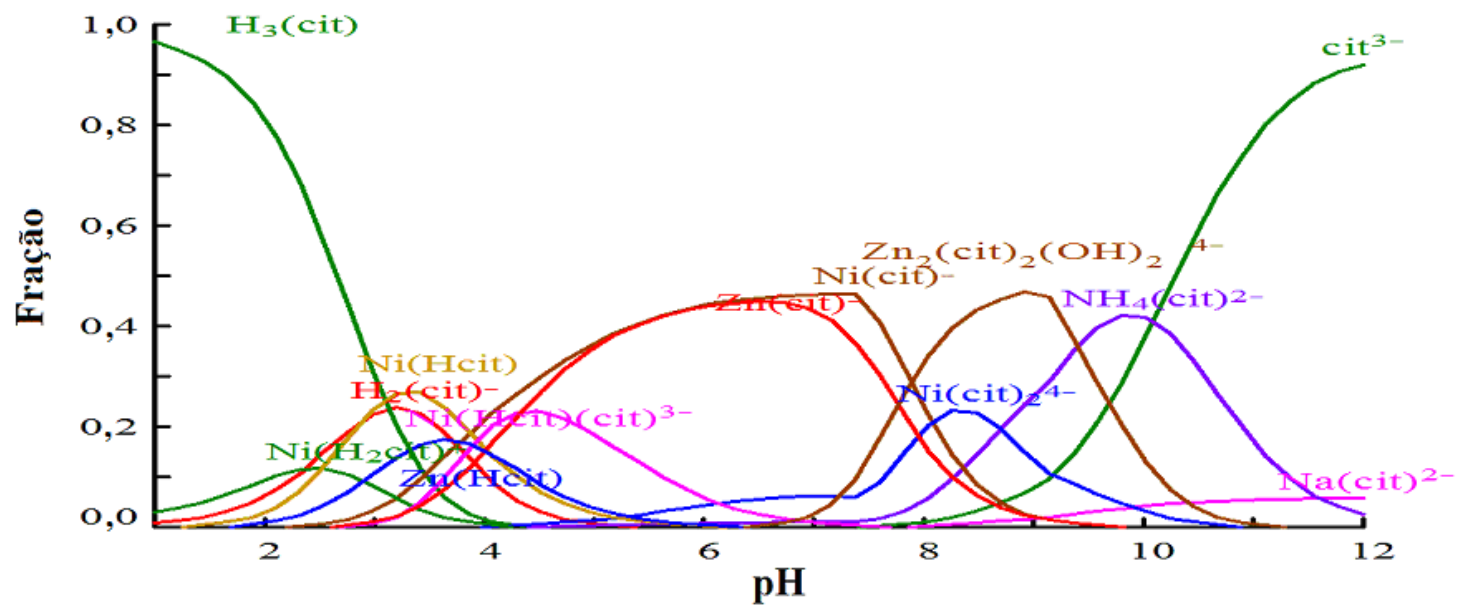

Observa-se da Figura 1 que em torno de $\mathrm{pH}$ 6, são formados em grande quantidade complexos iônicos de citrato de ambos metais que se deseja depositar. Dessa forma, determinou-se $\mathrm{pH}$ 6, como o $\mathrm{pH}$ de trabalho dos experimentos.

\section{RESULTADOS E DISCUSSÃO}

A matriz de planejamento apresentada na Tabela 2 lista os ensaios de eletrodeposição na ordem padrão, e mostra as eficiências de deposição obtidas em cada um deles. O gráfico de Pareto apresentado na Figura 2 com um nível de confiança de 95\% para o cálculo dos efeitos lineares principais e os efeitos de primeira ordem para valores absolutos, levando-se em consideração a eficiência de deposição foi obtidos a partir dos dados da Tabela 2. As barras representam a magnitude de cada efeito e a linha tracejada que corresponde ao valor de $\mathrm{p}=$ 0,05 indica significância estatística para efeitos que a ultrapassem. Com essa confiança podese afirmar que a densidade de corrente foi a variável que obteve maior influência estatisticamente significativa sobre a eficiência de deposição.

Tabela 2 - Planejamento fatorial $2^{2}$ em função da eficiência de deposição

\begin{tabular}{|c|c|c|c|}
\hline Exp. & $\mathrm{I}\left(\mathrm{mA} / \mathrm{cm}^{2}\right)$ & Agitação (rpm) & Eficiência (\%) \\
\hline 1 & -1 & -1 & 88,27 \\
\hline 2 & +1 & -1 & 18,50 \\
\hline 3 & -1 & +1 & 93,04 \\
\hline 4 & +1 & +1 & 25,30 \\
\hline $5(\mathrm{C})$ & 0 & 0 & 49,53 \\
\hline $6(\mathrm{C})$ & 0 & 0 & 47,80 \\
\hline $7(\mathrm{C})$ & 0 & 0 & 39,28 \\
\hline
\end{tabular}


Ainda de acordo com o gráfico de Pareto (Figura 2) observa-se que a densidade de corrente influencia a eletrodeposição quando se apresenta em valores menores, devido ao valor negativo atribuído à barra correspondente a este efeito.

Figura 2 - Diagrama de Pareto dos efeitos da eficiência de eletrodeposição

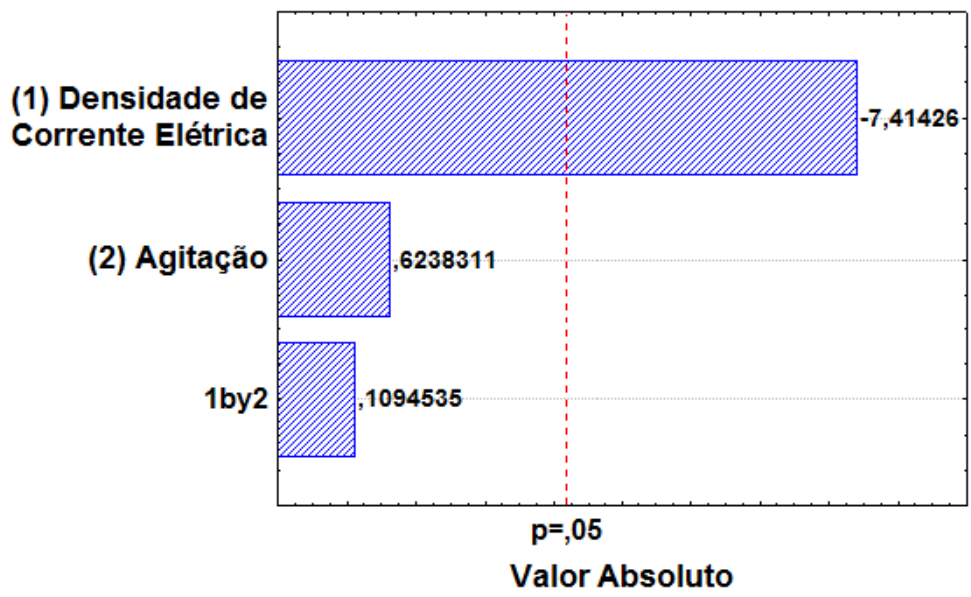

O modelo fornecido pelo aplicativo STATISTICA 7 ajusta-se aos dados experimentais de eficiência de deposição do processo de eletrodeposição da liga de $\mathrm{Zn}-\mathrm{Ni}$, conforme a Equação 2. O ajuste do modelo aos dados foi satisfatório, pois, o coeficiente de regressão $\left(\mathrm{R}^{2}\right)$ apresentou valor de 0,9486 .

$$
y=120,43-1,72 \cdot I
$$

sendo, y é a eficiência de deposição (\%), I é a densidade de corrente elétrica $\left(\mathrm{mA} / \mathrm{cm}^{2}\right)$ e esta equação é válida para o intervalo de estudo: $10 \mathrm{~mA} / \mathrm{cm}^{2}<\mathrm{I}<50 \mathrm{~mA} / \mathrm{cm}^{2}$.

A Figura 3 apresenta a média dos pontos experimentais e modelo ajustado (linha reta) para eficiência de deposição. Observa-se que a linearidade dos experimentos é pouco desviada pelos experimentos do nível (0).

Figura 3 - Valores preditos de eficiência versus valores observados

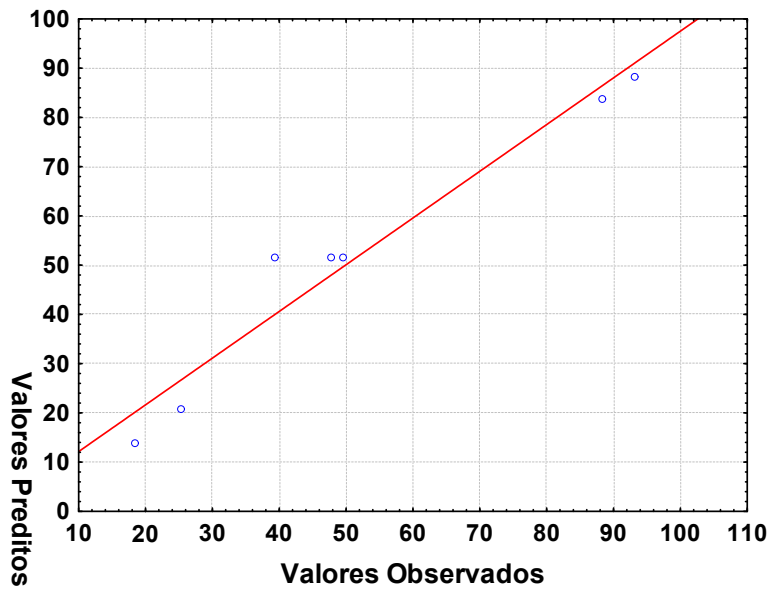


É possível observar o efeito da densidade de corrente versus agitação mecânica na superfície de resposta apresentada na Figura 4, onde a resposta corresponde à eficiência de deposição. De acordo com a superfície de resposta da Figura 4, verifica-se que com a maior densidade de corrente (I) é obtida a máxima eficiência de deposição, 93,04\%, sendo a variação da agitação pouca significativa na resposta.

Figura 4 - Superfície de resposta da eficiência de deposição em função da densidade de corrente (I) e da agitação mecânica

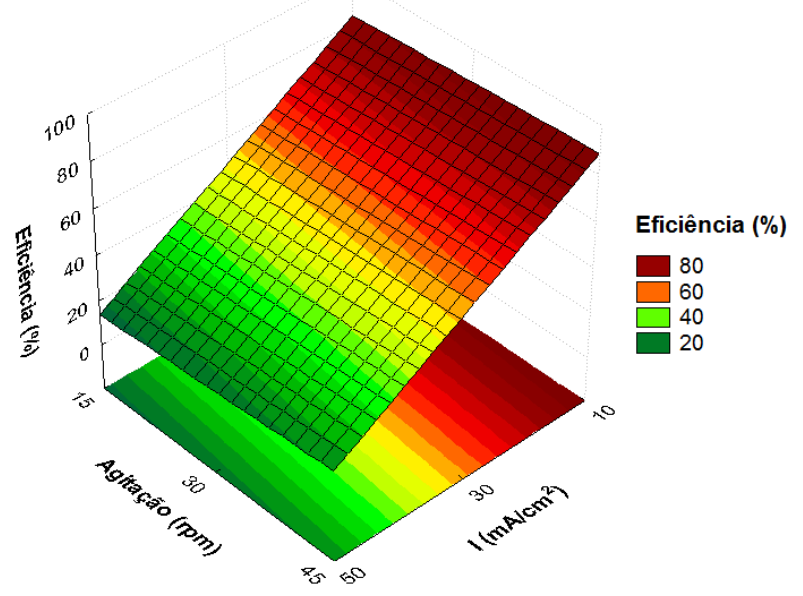

A aparência do depósito variou a cada ensaio, tendo o experimento 3 apresentado não só a melhor eficiência de deposição, mas também brilho e aderência nas bordas, como é possível verificar na Figura 5.

Figura 5 - Depósito da liga de Zn-Ni em substrato de cobre obtido por processo de eletrodeposição nas condições do Experimento 3

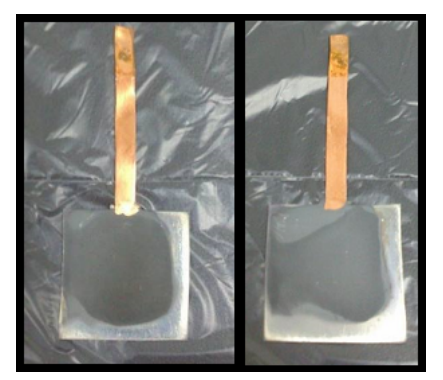

\section{CONCLUSÃO}

Para a liga de $\mathrm{Zn}-\mathrm{Ni}$, os melhores valores dos parâmetros agitação mecânica e densidade de corrente elétrica num sistema de eletrodeposição à temperatura ambiente, $\mathrm{pH}$ 6,0 e concentrações do banho eletrolítico constantes foram $10 \mathrm{~mA} / \mathrm{cm}^{2}$ e $45 \mathrm{rpm}$, respectivamente. O valor da eficiência de deposição máxima obtida foi de $93,04 \%$. As análises estatísticas mostraram que a variável que mais influenciou o processo de eletrodeposição foi a densidade de corrente elétrica. Os parâmetros trabalhados neste estudo foram otimizados em um depósito com brilho e aderência nas bordas do substrato de cobre. 


\section{REFERÊNCIAS}

ALFANTAZI, A. M.; PAGE, J.; URB, U. Pulse plating of Zn-Ni alloy coatings. J. Appl. Eletrochem. 26, p. 1225-1234, 1996.

ANICAI, L.; SITEAVU, M.; GRUNWALD, E. Corrosion behavior of zinc and zinc alloy deposition. Corros. Prevention Control 39 (4), p. 89-93, 1992

BARROS NETO, B.; SCARMINIO, I. S.; BRUNS, R. E. Planejamento e otimização de experimentos, 2. Ed., Campinas - SP: Editora da Unicamp, 1996.

BELTOWSKA-LEHMAN, E.; INDYKA, P. Kinects of Ni-Mo electrodeposition from Ni-rich citrate baths. Journal Elsevier, Thin Solid Films 520, p. 2046-2051, 2012.

BELTOWSKA-LEHMAN, E.; OZGA, P.; SWIATEK, Z.; LUPI, C. Cryst. Eng. 5, p. 335, 2002.

BROOMAN, E. W. Alternatives to cadmium coating for electrical/electronic applications, Plating Surf. Finish. 80, p. 29-35, 1993.

CROTTY, D.; Zinc alloy plating for the automotive industry. Met. Finish. 94, p. 54-58, 1996.

FRATESI, R.; ROVENTI, G. Surface and Coatings Technology 82, p.158, 1996.

GOMEZ, E.; PELLICER, E.; VALLES, J. Electroanal. Chem. 517, p.109, 2001.

HEGDE, A. C.; VENKATAKRISHNA, K.; ELIAZ, N. Electrodeposition of Zn-Ni, Zen-Fe and Zn-Ni-Fe alloys. Journal Elsevier, Surface and Coatings Technology 205, p.20312041, 2010.

LIN, Y. P.; SELMAN, J. R. Journal Electrochem. Soc. 140, p. 1299, 1993.

MIYOSHI, Y. Journal ISIJ Int. 31, p.1, 1991.

RIEDEL, Proteccion contra la corrosion mediante recumbrimientos de aleaciones de cinc depositados eletrolíticamente, Pinturas y Acabados Industriales 30 (162), p. 13-16, 1988.

RAMANAUSKAS, R. Appl. Surf. Sci. 153, p. 53, 1999.

SHORT, N. R.; ZHOU, S.; DENNIS, J. K. Surface and Coatings Technology 79, p.218, 1996.

WRIGHT, D. A.; GAGE, N.; WILSON, B. A. Zinc-nickel electroplate as a replacement for cadmium on high-strength steels, Plating Surf. Finish. 81, p 18-21, 1994.

\section{AGRADECIMENTOS}

À FAPESP pelo auxílio financeiro. 